\title{
First series of minimally invasive, robot-assisted tracheobronchoplasty with mesh for severe tracheobronchomalacia
}

\author{
Richard Lazzaro, MD, ${ }^{a}$ Byron Patton, MD, ${ }^{a}$ Paul Lee, MD, ${ }^{b}$ Jason Karp, MD, ${ }^{c}$ Efstathia Mihelis, PA-C, ${ }^{a}$ \\ Sohrab Vatsia, MS, ${ }^{a}$ and Samuel Jacob Scheinerman, MD ${ }^{a}$
}

\section{ABSTRACT}

Objective: Tracheobronchomalacia is a progressive, debilitating disease with limited treatment options. Open tracheobronchoplasty (TBP) is an accepted surgical option for management of severe tracheobronchomalacia. This study examined the outcomes of the first reported series of robot-assisted TBP (R-TBP).

Methods: We retrospectively reviewed the records of patients with clinical suspicion for tracheobronchomalacia who had dynamic computed tomography scan and subsequent R-TBP from May 2016 to December 2017.

Results: Four hundred thirty-five patients underwent dynamic computed tomography scan for suspicion of tracheobronchomalacia. Of this group, 42 patients underwent R-TBP. In the surgery group, the median age was 66 years (interquartile range, 39-72 years) and there were 30 women (71\%). Respiratory comorbidities included asthma (88\%) and chronic obstructive pulmonary disease $(52 \%)$. The median operative time was 249 minutes (interquartile range, 266-277 minutes). Median hospital length of stay was 3 days (interquartile range, 2-4.75 days), and there were 19 postoperative complications (11 minor and 8 major). There were no mortalities at 90 days. Comparison of preoperative and postoperative pulmonary function testing demonstrated improvement in forced expiratory volume at 1 second by $13.5 \%(P=.01)$, forced vital capacity by $14.5 \%(P<.0001)$, and peak expiratory flow rate by $21.0 \%(P<.0001)$. Quality of life questionnaires also showed improvement with $82 \%$ reporting overall satisfaction with the procedure.

Conclusions: R-TBP can be performed with low morbidity and mortality. Early follow-up reveals significant improvement in pulmonary function testing and high patient satisfaction when compared with preoperative baseline. Long-term follow-up is needed to demonstrate the durability of R-TBP and substantiate its role in the management of patients with symptomatic, severe tracheobronchomalacia. (J Thorac Cardiovasc Surg 2019;157:791-800)

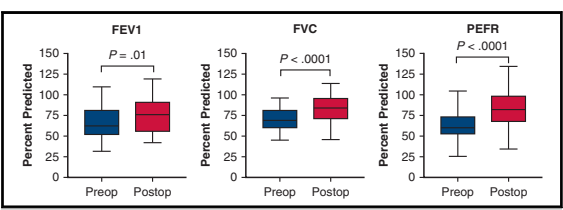

Improvement of pulmonary function tests following robot-assisted tracheobronchoplasty.

\section{Central Message}

Robotic tracheobronchoplasty for adult tracheobronchomalacia is safe; associated with significant improvement in FEV1, FVC, and PEFR; and can further the benefits of open tracheobronchoplasty.

\section{Perspective}

Robotic tracheobronchoplasty is safe and associated with early improvement in FEV1, FVC, and PEFR. The robot-assisted platform is ideal for the minimally invasive surgical approach to tracheobronchomalacia. The surgical procedure requires a standard approach to dissection that can be taught and learned by a wide breadth of thoracic surgeons. The robotic platform is ideal to further the adoption of surgical treatment of tracheobronchomalacia in adults.

See Editorial Commentary page 801.

\footnotetext{
From the ${ }^{\text {a Department }}$ of Cardiothoracic Surgery, Lenox Hill Hospital, New York, NY; ${ }^{b}$ Department of Cardiothoracic Surgery, Long Island Jewish Medical Center, New Hyde Park, NY; and ${ }^{c}$ Department of Pulmonary Medicine, North Shore University Hospital, Manhasset, NY.

Read at the 98th Annual Meeting of The American Association for Thoracic Surgery, San Diego, California, April 28-May 1, 2018.

Received for publication Jan 15, 2018; revisions received July 13, 2018; accepted for publication July 22, 2018.

Address for reprints: Richard Lazzaro, MD, Department of Cardiothoracic Surgery, Lenox Hill Hospital, 130 E 77th St, New York, NY 10075 (E-mail: rlazzaro@ northwelll.edu).

0022-5223

Copyright (C) 2018 by The American Association for Thoracic Surgery. Published by Elsevier Inc. This is an open access article under the CC BY-NC-ND license (http:// creativecommons.org/licenses/by-nc-nd/4.0/).

https://doi.org/10.1016/j.jtcvs.2018.07.118
}

Tracheobronchomalacia (TBM) is characterized by weakness of the trachea and 1 or both of the mainstem bronchi. TBM commonly manifests through an acquired etiology; that is, secondary to chronic obstructive

\begin{tabular}{l}
\hline Scanning this QR code will \\
take you to the article title \\
page to access supplementary \\
information. To view the \\
AATS Annual Meeting Web- \\
cast, see the URL next to the \\
webcast thumbnail.
\end{tabular}




\section{Abbreviations and Acronyms \\ $6 \mathrm{MWT}=$ Six-Minute Walk Test \\ COPD $=$ chronic obstructive pulmonary disease \\ $\mathrm{CT}=$ computed tomography \\ FEV1 = forced expiratory volume at 1 second \\ GERD $=$ gastroesophageal reflux disease \\ $\mathrm{O}-\mathrm{TBP}=$ open tracheobronchoplasty \\ PFT = pulmonary function test \\ $\mathrm{R}-\mathrm{TBP}=$ robot-assisted tracheobronchoplasty \\ SGRQ $=$ St George's Respiratory Questionnaire \\ $\mathrm{TBM}=$ tracheobronchomalacia \\ VATS $=$ video-assisted thoracic surgery}

pulmonary disease (COPD), relapsing polychondritis, tracheotomy, or chronic inhaled corticosteroid use. ${ }^{1-3}$ Pathologically, severe TBM leads to atrophy of the posterior airway membrane and complete or nearcomplete collapse of the anterior tracheobronchial cartilage wall, ultimately attenuating dynamic airflow. ${ }^{4}$ As a result, patients exhibit dyspnea, persistent cough, and are often predisposed to pneumonia and other respiratory infections. ${ }^{5}$ Refractory and intractable symptoms may prompt further investigation with dynamic imaging and bronchoscopy leading to a diagnosis of TBM. However, because there are no published guidelines for clinical identification of TBM, a high degree of suspicion is needed. It has been estimated that the overall incidence of TBM is $5 \%$ to $10 \%$ in the general population, and a cutoff of $\geq 70 \%$ narrowing on forced expiration has been recognized to diagnose TBM. ${ }^{6}$ Severe TBM has been defined as complete or near-complete collapse of the trachea, demonstrated by functional bronchoscopy and dynamic computed tomography (CT) scanning. ${ }^{7}$

Treatment of the disease consists of tracheobronchial stenting of the airway and open tracheobronchoplasty (O-TBP). These techniques stabilize the airway in patients by restoring more normal airway geometry. ${ }^{8,9}$ Both self-expanding metallic stents and silicone $\mathrm{Y}$ stents have been studied previously with indication of some temporary relief of symptoms. Expandable metal stents preserve mucociliary function and can be placed with relative simplicity but may be difficult to remove secondary to granulation tissue in inflamed airways. ${ }^{10}$ Silicone Y stents may improve dyspnea and quality of life ${ }^{3}$; however, complications such as mucus plugging, foreign body irritation with cough, and stentrelated infections may occur. ${ }^{10}$ Stenting has been established as a short-term surrogate for O-TBP and may help determine candidates for surgical treatment. However, stents are not effective as a long-term solution. ${ }^{11}$

The goal of O-TBP is to achieve stabilization of the trachea and both mainstem bronchi by posterior membrane plication and splinting of the posterior cartilage using polypropylene mesh. ${ }^{7}$ TBP is performed through right posterolateral thoracotomy, with exposure of the intrathoracic trachea, bilateral main bronchi, and bronchus intermedius. Mesh is sutured to the posterior membrane and cartilage in a partial thickness fashion to avoid entering the airway lumen, thereby restoring normal airway geometry and plication of the redundant membrane. ${ }^{7}$ Wright and colleagues ${ }^{8}$ demonstrated significant improvement in forced expiratory volume at 1 second (FEV1), forced vital capacity, and peak expiratory flow rate in 14 patients using this technique. However, the largest published series of 63 patients by Gangadharan and colleagues ${ }^{7}$ showed no improvement in FEV1. This series also showed improvement in quality of life, respiratory symptoms, and functional status, but the authors acknowledge that these subjective measures may have confounding variables making definitive conclusions difficult. Consequently, "the search for an objective measurement of improvement remains the Holy Grail of intervention for TBM.," 11

Robot-assisted thoracoscopic surgery provides a stereoscopic, high-definition view with enhanced degrees of freedom and scaled motion. Multiple studies show low morbidity and mortality for other robot-assisted thoracic procedures such as lobectomy. ${ }^{12,13}$ In 2015, Lazar and colleagues ${ }^{14}$ substantiated the feasibility of robotic-assisted bilateral bronchoplasty for the treatment of TBM. The benefits (eg, decreased morbidity) of minimally invasive surgery have been elucidated over the past decades through numerous publications. The rationale for robot-assisted TBP (R-TBP) is that application of minimally invasive technology to an established, but complex, open technique may allow the surgeon to perform the same operation with decreased mortality associated with minimally invasive surgery. The purpose of this study was to evaluate patient demographic characteristics, complications, and surgical outcomes of minimally invasive R-TBP for the treatment of symptomatic patients with severe TBM.

\section{PATIENTS AND METHODS}

This retrospective study was approved by the Northwell Health Institutional Review Board. Study data were collected and managed using Research Electronic Data Capture electronic data capture tools hosted at Northwell Health. ${ }^{15}$ Research Electronic Data Capture is a secure, web-based application designed to support data capture for research studies, providing an intuitive interface for validated data entry, audit trails for tracking data manipulation and export procedures, automated export procedures for seamless data downloads to common statistical packages, and procedures for importing data from external sources.

Inclusion criteria were: all patients with clinical suspicion of TBM who underwent dynamic CT scan from May 2016 to December 2017 at a Northwell Health facility. TBM was defined as $\geq 50 \%$ collapse of the airway by dynamic CT. The charts of all patients with evidence of TBM on dynamic CT scan were reviewed. Patients were excluded from this study if they were unable to complete dynamic CT scanning or were no longer alive at time of chart review.

Severe TBM was defined as $>90 \%$ collapse on awake bronchoscopy. R-TBP inpatient and outpatient charts were reviewed to assess for any complications within the first 90 days after surgery and were graded based 


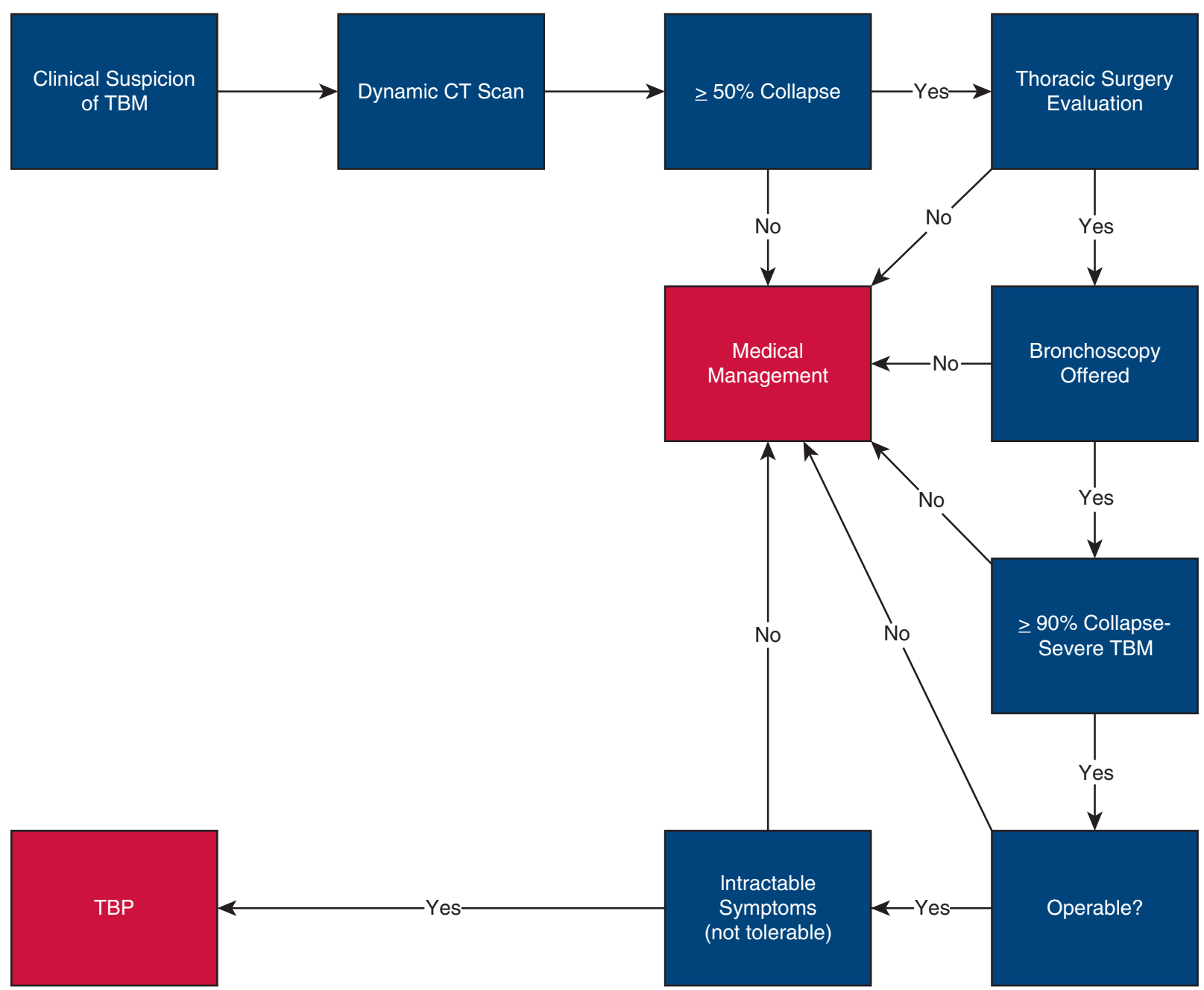

FIGURE 1. Evaluation and management of patients suspected of having tracheobronchomalacia (TBM). Patients with clinical suspicion of TBM are referred for dynamic computed tomography $(C T)$ scan according to established Northwell Health Imaging protocol. Patients who exhibit collapse of the tracheobronchial tree $\geq 50 \%$ on CT are evaluated by a member of the thoracic surgery team. Surgical candidates for robot-assisted tracheobronchoplasty $(T B P)$ who demonstrate persistent, severe symptoms are offered bronchoscopy. If bronchoscopic findings confirm $>90 \%$ collapse of the airway, and if the airway is deemed operable for relief of intractable symptoms, patients undergo robot-assisted TBP. If patients do not meet any of these surgical inclusion criteria, they are treated by medical management.

on Clavien-Dindo classification. ${ }^{16}$ Preoperative and postoperative pulmonary function testing, St George's Respiratory Questionnaire ${ }^{17}$ (SGRQ), and Six-Minute Walk Test ${ }^{18}$ (6MWT) data were collected and analyzed for the surgical group. A 5-point postoperative Likert scale was used to assess subjective change in cough, shortness of breath, ability to manage respiratory infections, ability to move air with breathing, and overall satisfaction with the procedure. No surgical patients were excluded from this study.

\section{Diagnosis}

Pulmonary medicine team members evaluated each patient for symptoms consistent with TBM, including progressive dyspnea, chronic severe cough, and recurrent pulmonary infections. Comorbid conditions were actively managed by primary care physicians as well as specialists, and included gastroesophageal reflux disease (GERD), sinus conditions, COPD, asthma, and obstructive sleep apnea. Asthma ${ }^{19}$ and $\mathrm{COPD}^{20}$ were defined in accordance with American Thoracic Society guidelines,
GERD was diagnosed by symptomatology of esophageal injury secondary to gastric content reflex, and cardiovascular disease encompassed arrhythmia, coronary artery disease, and heart failure. Active smokers were counseled on smoking cessation. If symptoms persisted despite maximal medical therapy, patients were referred for dynamic airway screening CT scan, which was performed according to a protocol. This protocol included a full chest CT scan on inspiration followed by ultra-low-dose CT during exhalation and cine images attained during forced exhalation. A diagnosis of TBM or findings suggestive of TBM was rendered by Board-certified radiologists. Patients with radiographic findings of $\geq 50 \%$ collapse of the tracheobronchial tree were referred for thoracic surgical evaluation. Patients with persistent, severe symptoms underwent awake bronchoscopy. Severe symptoms were defined as those that negatively influence a patient's everyday life, frequent hospitalizations with pulmonary infection, or escalating medications such as steroids with no improvement. Bronchoscopic findings demonstrating $>90 \%$ collapse of the airway on exhalation was used to confirm the diagnosis of severe TBM (Figure 1). 


\section{Stent Trial}

Patients with intractable symptoms who were diagnosed with severe TBM by dynamic CT scan and awake bronchoscopy, underwent tracheobronchial stent trial. The initial 11 patients underwent stent trial with self-expanding nitinol stents of the trachea, bronchus intermedius, and left main bronchus. Eight of 11 patients had improvement in symptoms and underwent R-TBP 2 weeks after stent explantation. The remaining 3 patients had increased cough and noncardiac chest pain during the stent trial that resolved following stent removal. One of these 3 , and the last of our stent trial patients, required stent removal 48 hours following initial placement secondary to fever and hypotension, which resolved soon after removal. However, after recovery from the stent trial, symptoms returned, and all 3 patients elected to undergo R-TBP for progressive, symptomatic, severe TBM. Due to these stent complications, the ability of a stent trial to assess symptom improvement and help patient selection was determined to be inadequate and no further stent trials were performed on subsequent patients. Patients with severe symptoms not responsive to medical therapies and severe TBM on bronchoscopy ( $>90 \%$ collapse) were offered R-TBP.

\section{Surgical Management}

The surgical approach requires general anesthesia, double lumen endotracheal intubation, and robot-assisted video-assisted thoracic surgery, utilizing 4 robotic arms/ports with 1 additional $12-\mathrm{mm}$ port for the assistant. The patient is positioned in the left lateral decubitus position with the da Vinci XI (Intuitive Surgical Inc, Sunnyvale, Calif) robot brought in from the right side of the patient and the boom rotated clockwise to position it over the head of the patient. The robot is docked to the ports and carbon dioxide insufflation (to aid resorptive atelectasis) to a pressure of 8 is initiated as instruments are inserted. With gentle traction, utilizing a sponge with the fourth arm of the robot, the deflated right lung is retracted anteriorly and medially. The azygos vein is dissected and divided to facilitate exposure of the entirety of the intrathoracic trachea and mainstem bronchi. Bipolar energy is used for dissection, which is carried out anterior to the esophagus from the level of the thoracic inlet to the inferior pulmonary vein. With continued gentle anteromedial traction on the deflated right lung, the subcarinal space is visualized. Routinely, the contralateral superior pulmonary vein is also identified. Subcarinal lymph nodes are either dissected away from the airway or resected to expose the cartilaginous rings of the left and right bronchi. Posterior splinting left main bronchoplasty is performed with a $5 \times 1.4 \mathrm{~cm}$ polypropylene braided mesh. Three rows of partial thickness sutures (4-0 Vicryl, RB -1; Ethicon Inc, Somerville, NJ) are utilized to secure the mesh to the posterior cartilage. The lateral sutures are placed several $\mathrm{mm}$ away from the edge of the mesh and placed partial thickness through the posterior cartilage and back through the mesh. Sutures are placed from distal to proximal, and each individual suture incorporates a distinct separate bronchial cartilage. These sutures are tied, securing the mesh to the airway. Horizontal mattress sutures are then placed through the mesh, followed by posterior membrane, and then back through the mesh. The middle row of horizontal mattress sutures pleat the posterior membrane to the mesh. Before securing the pleating sutures, the medial sutures are placed through the mesh, partial thickness through the medial posterior cartilage and back through the mesh. Cartilaginous sutures are tied before securing the pleating sutures. Occasionally, proximal bronchoplasty requires 4 rows of sutures. The effective bronchial width of the mesh is $10 \mathrm{~mm}$. An $8 \times 2.2 \mathrm{~cm}$ polypropylene mesh is secured to the trachea with 4 rows of sutures for an effective tracheal width of $16 \mathrm{~mm}$. Sutures are placed several millimeters away from the edge of the mesh and placed partial thickness through the posterior cartilage of the left aspect of the trachea, and back through the mesh. Sutures are placed from proximal to distal, and each individual suture incorporates a distinct separate tracheal cartilage. These sutures are tied, securing the mesh to the airway. Two columns of horizontal mattress sutures are then placed through the mesh, followed by posterior membrane, and then back through the mesh. Horizontal mattress sutures are used for the middle 2 rows to more effectively pleat the posterior membrane. Before securing the pleating sutures, the medial sutures are place through the mesh, partial thickness through the right-sided posterior tracheal cartilage and back through the mesh. Cartilaginous sutures are tied before securing the pleating sutures. Repair of the right mainstem bronchus and bronchus intermedius is similar to repair of the left mainstem bronchus with the same size mesh and effective bronchial width. Care must be taken to avoid narrowing the right upper lobe bronchus. All sutures are placed at partial thickness to avoid potential air leak and risk of contamination of the mesh. Bronchoscopy is performed before completion of the procedure to assess the repair as well as patency of all lobar and segmental bronchi. A solitary $24 \mathrm{Fr}$ chest tube is placed. For pain control, intercostal nerve blocks as well as intravenous acetaminophen and ketorolac were used. The patients are extubated in the operating room and transferred to the intensive care unit (Video 1).

\section{Statistical Analysis}

Continuous data are summarized as median and interquartile range (IQR). Preoperative and postoperative measurements of pulmonary function tests (PFTs), 6MWTs, and SGRQ quality of life questionnaires were compared using Wilcoxon signed-rank paired analyses, given the nonparametric data distribution.

\section{RESULTS}

Between September 2016 and November 2017, 435 patients underwent dynamic CT scan for clinical suspicion of TBM. Of this group, 183 had $>50 \%$ collapse by CT. One hundred forty-five were evaluated by thoracic surgery and 42 consecutive patients underwent R-TBP for severe symptomatic TBM (Figure 2).

The median age of the surgical group was 66 years (IQR, 59-72 years) with 30 female patients $(71 \%)$. The median body mass index was 29 (IQR, 27-34). There were 2 patients $(4 \%)$ with a recent history of smoking $(<1$ year before R-TBP). Patients exhibited respiratory comorbidities, including asthma (88\%) and COPD (52\%) and also presented concurrently with GERD (85\%),

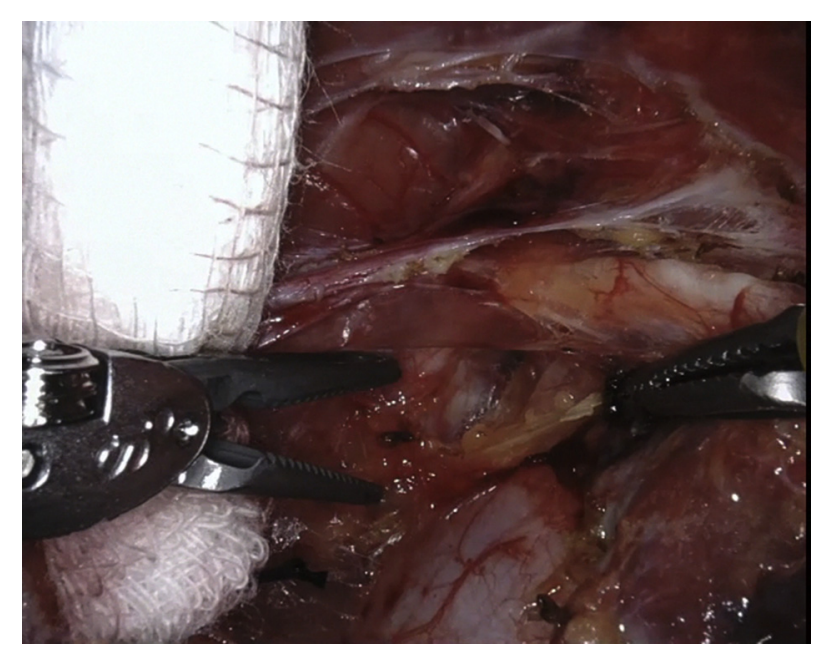

VIDEO 1. Robotic tracheobronchoplasty technique. Video available at: https://www.jtcvs.org/article/S0022-5223(18)32935-0/fulltext. 


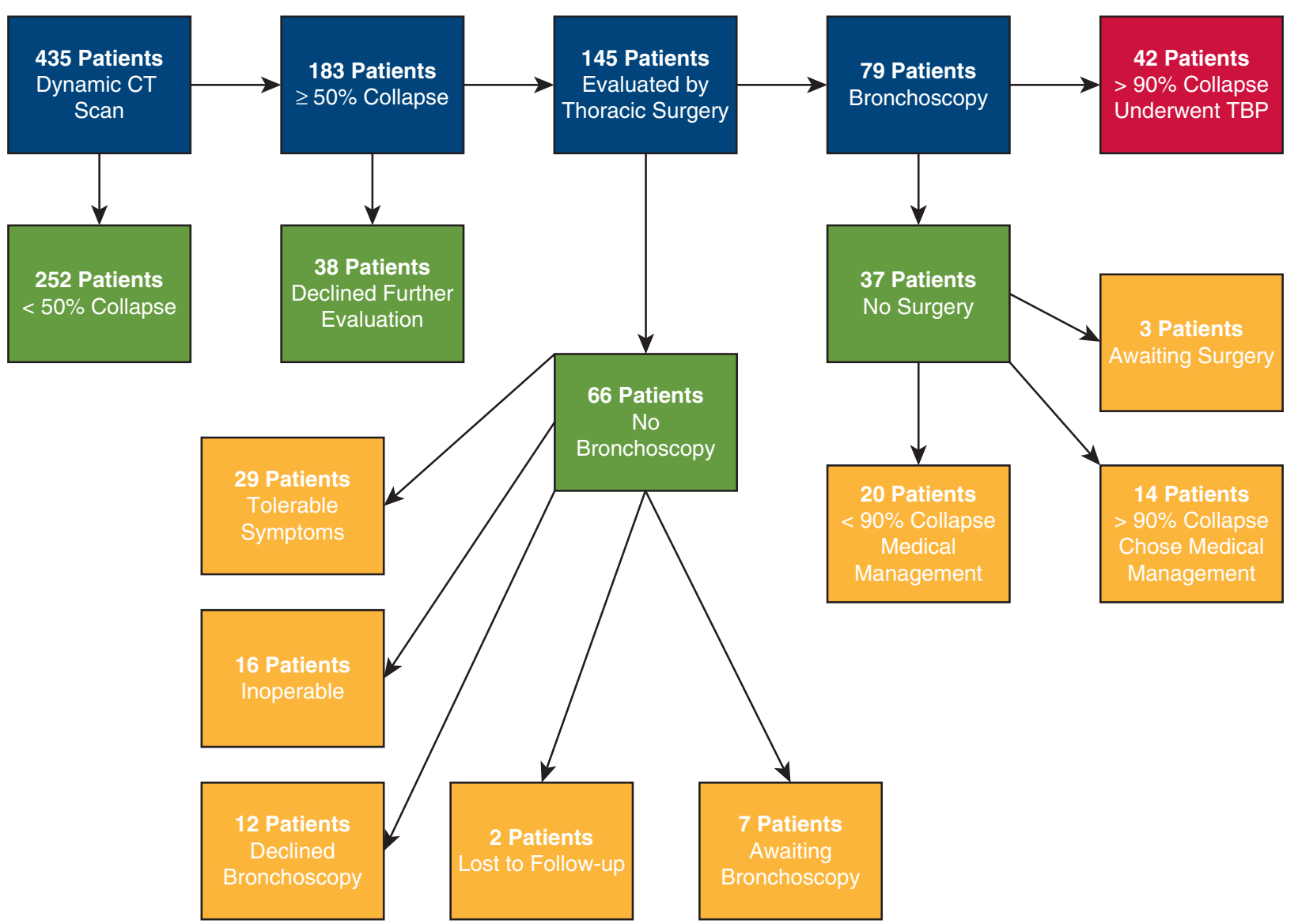

FIGURE 2. Lenox Hill Northwell Health tracheobronchomalacia (TBM) program. Four hundred thirty-five patients underwent dynamic computed tomography $(C T)$ scan for clinical suspicion of TBM. Of this group, 183 had $>50 \%$ collapsed, as observed by CT. One hundred forty-five patients were evaluated by members of the thoracic surgery team and 42 consecutive patients underwent robot-assisted tracheobronchoplasty (TBP) for severe symptomatic TBM.

hypertension (52\%), diabetes mellitus $(26 \%)$, and cardiovascular disease (14\%). Six patients (14\%) had prior chest surgery, including ipsilateral video-assisted thoracic surgery (VATS) right upper lobectomy, open bilobectomy, VATS left lower lobectomy, VATS left upper lobe trisegmentectomy, left VATS wedge resection, and coronary artery bypass surgery. The median operative time was 249 minutes (IQR, 226-277 minutes) and no blood transfusions were necessary. Chest tubes were removed at a median of 1 day after surgery (IQR, 1-1 day). The median hospital length of stay was 3 days (IQR, 2-4.75 days) and median length of intensive care unit stay was 1 day (IQR, 1-2 days).

There were a total of 19 postoperative complications within the first 90 days, including 11 minor complications $(26 \%)$ and 8 major complications $(19 \%)$ as defined by Clavien-Dindo classification schema. ${ }^{16}$ Four complications were Grade I (1 pneumothorax and 3 subcutaneous emphysema) that required no intervention. Seven Class II complications were all infectious in etiology (4 bronchitis and 3 pneumonia) and treated with antibiotics. Of the major complications, 4 were Class IIIa (2 chest tube insertions for pneumothorax and 2 infraclavicular blowholes for subcutaneous emphysema) and did not require general anesthesia for intervention. Two complications were Grade IIIb and required general anesthesia for intervention. One patient had VATS evacuation of hemothorax after chest tube insertion on postoperative day 2. Although not within the first 90 days, another patient required revision surgery for persistent symptoms on postoperative day 125 . Two patients $(4 \%)$ had unplanned return to an intensive care unit (Grade IV): 1 for bronchoscopy and the other for arrhythmia (tachy-brady syndrome). There were no reintubations, no tracheostomies, and no mortalities at 90 days.

Forty patients had complete sets of preoperative and postoperative PFTs in the surgical group. Postoperative PFTs were performed at a median of 4 months (IQR, 2-6 months). The median preoperative percent predicted FEV1 was $62.50 \%$ (IQR, $51.25 \%-82.00 \%$ ) and was $76.00 \%(\mathrm{IQR}, 55.25 \%-91.75 \%)$ after surgery $(P=.01)$ (Figure 3, A). The median preoperative percent 

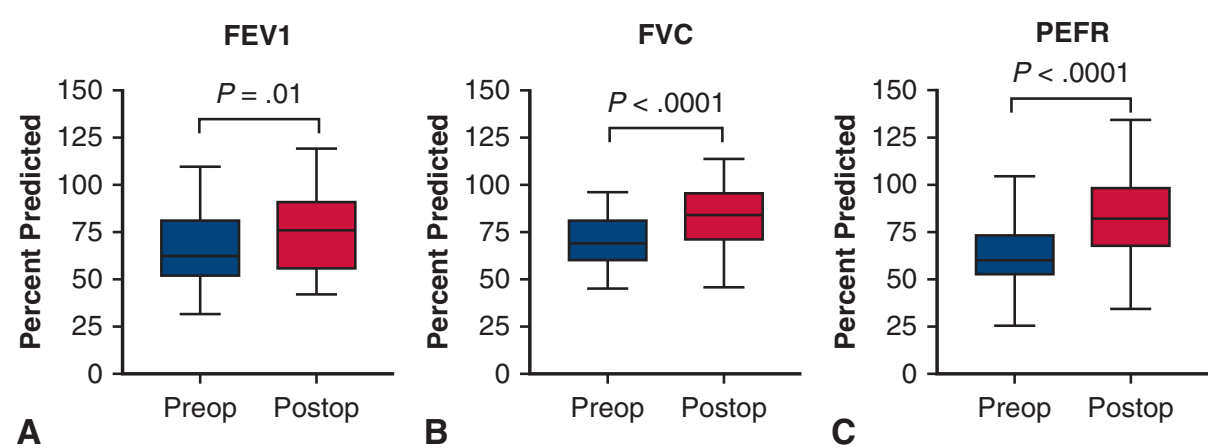

FIGURE 3. Median percent predicted lung function before and after robot-assisted tracheobronchoplasty. A, Forced expiratory volume in 1 second (FEV1). B, Forced vital capacity $(F V C)$. C, Peak expiratory flow rate $(P E F R)$. The median preoperative percent predicted FEV1 was $62.50 \%$ (interquartile range [IQR], $51.25 \%-82.00 \%)$ and was $76.00 \%$ (IQR, 55.25\%-91.75\%) after surgery $(P=.01)$. The median preoperative percent predicted FVC was $69.50 \%$ (IQR, $59.25 \%-82.25 \%$ ) and improved to $84.00 \%$ (IQR, 70.50\%-96.25\%) following operation $(P<.0001$ ). The median preoperative percent predicted PEFR was $60.00 \%$ (IQR, $52.00 \%-73.25 \%$ ) and rose to $81.00 \%$ (IQR, 67.25\%-101.75\%) after surgery $(P<.0001)$. The boxes indicate the IQR, with the upper boarder and lower boarder of the box indicating the upper and lower quartiles, respectively. Whiskers represent the minimum and maximum values. The central line demonstrates the median $(n=40$ patients per group).

predicted forced vital capacity was $69.50 \%$ (IQR, $59.25 \%$ $82.25 \%$ ) and improved to $84.00 \%$ (IQR, $70.50 \%-96.25 \%$ ) following operation $(P<.0001)$ (Figure 3, B). The median preoperative percent predicted peak expiratory flow rate was $60.00 \%$ (IQR, $52.00 \%-73.25 \%$ ) and rose to $81.00 \%$ (IQR, 67.25\%-101.75\%) after surgery $(P<.0001)$ (Figure 3, $C$ ). The percent change from preoperative pulmonary function per patient is illustrated in Figure 4.
In addition to undergoing pulmonary function testing perioperatively, 17 patients underwent $6 \mathrm{MWT}$ at a median of 5 months (IQR, 2-7 months) postoperatively; the median preoperative distance was $378.66 \mathrm{~m}$ (IQR, 255.11-389.63 m) and 380.49 $\mathrm{m}$ (IQR, 297.91-389.02 m) postoperatively $(P=.08)$. Additionally, 15 patients completed SGRQ quality-of-life measures at a median of 2 months (IQR, 1-4 months) postoperatively. The median

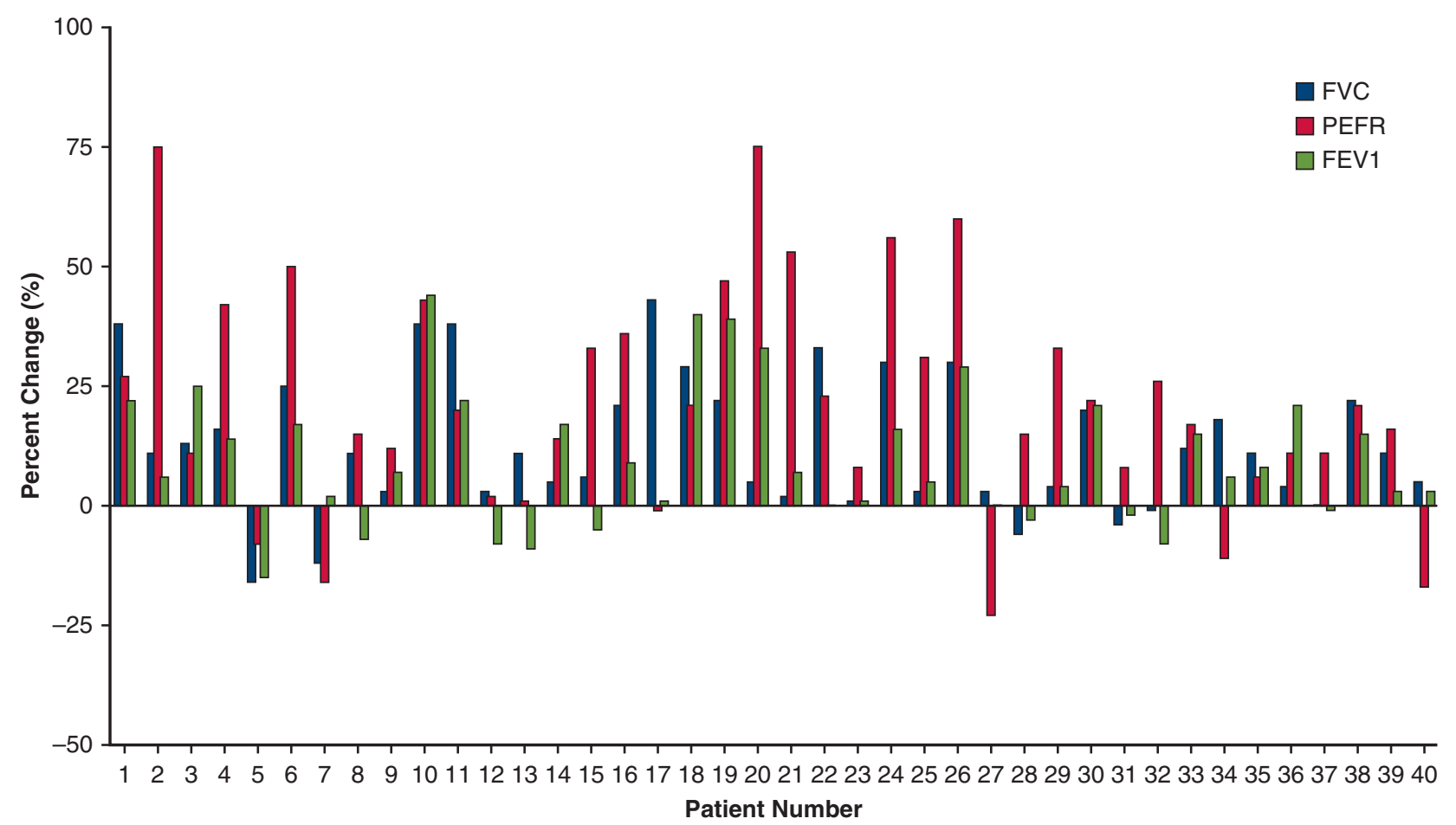

FIGURE 4. Percent change in percent predicted forced vital capacity $(F V C)$, peak expiratory flow rate (PEFR), and forced expiratory volume in 1 second (FEV1) by patient. Forty patients had complete sets of preoperative and postoperative PFTs. Percent predicted FEV1 (green bars), FVC (blue bars), and PEFR (red bars) were collected at a median of 4 months (interquartile range, 2-6 months) postoperatively. 
preoperative SGRQ score was 65.28 (IQR, 51.08-84.06) and decreased to 46.22 (IQR, 26.36-56.34) following R-TBP $(P=.002)$. Notably, the minimum clinically important difference defines the minimum change in a patient derived score that is meaningful for the patient and is generally agreed to be 4 units for SGRQ. ${ }^{21}$

Although long-term patient follow-up is presently ongoing, 35 patients completed a 5-point (Likert scale) survey assessing satisfaction with the R-TBP procedure at a median of 13 months postoperatively (IQR, 8.0-15.5 months). Twenty-four of 33 patients (72\%) who had symptomatic cough reported subjective improvement, 19 of 33 patients $(57 \%)$ who had symptomatic shortness of breath reported subjective improvement, 17 of 25 patients $(68 \%)$ reported subjective improvement of their ability to manage respiratory infections, 25 patients $(71 \%)$ reported subjective improvement of their ability to move air with breathing, and 29 patients $(82 \%)$ reported satisfaction with the overall results of the procedure.

\section{DISCUSSION}

This study shows that R-TBP is feasible and can be performed with significant improvement in PFTs and high overall patient satisfaction. The robotic platform allows emulation of an open approach by giving the surgeon improved visualization, flexibility of wristed instruments, and the precision to place dozens of sutures without injury to the airway through scaled motion. The significant increase in 3 PFT parameters indicates that the technical aspects of the repair are not compromised with the minimally invasive approach. In addition, $82 \%$ of patients reported overall satisfaction with the results. Although the interpretation of subjective data are difficult due to confounding variables, it is encouraging that many patients also had objective improvement.

In the 42 patients who underwent R-TBP, there were 11 minor complications and 8 major complications with only 1 patient requiring bronchoscopy postoperatively and another undergoing VATS for hemothorax after bedside chest tube insertion. One patient was discharged on postoperative day 2 but had revision 4 months after surgery for recurrent TBM. All patients were discharged home. There were no reintubations, no tracheostomies, and no deaths. Postoperative pain was controlled with anti-inflammatory medications and minimal narcotics. Aggressive pulmonary toilet and ambulation likely aided in avoiding pulmonary complications.

This study has limitations, including its retrospective nature and incomplete measurement of subjective change in symptoms by SGRQ. On a postoperative survey, most patients reported overall satisfaction, but not all symptoms improved to the same degree. Chronic cough was most improved with shortness of breath the least. Currently, we enroll all patients with TBM referred for thoracic surgery in a prospective study that includes not only PFTs, but also subjective measures such as SGRQ. Because all operations were performed over a short period of time, long-term follow-up will be necessary to establish the durability of the repair. In addition, all operations were performed at a single institution and similar outcomes would need to be reproduced to validate the technique and to establish it as a standard operative approach.

The future of TBP seems bright. TBM is a vastly underdiagnosed condition that has remained virtually untreated for decades. The prevalence of TBM has been estimated to be as high as $10 \%$ in the general population, ${ }^{22}$ but review of data from the Society of Thoracic Surgeons General Thoracic Surgery Database ${ }^{23}$ shows only 262 tracheoplasties performed from 2002 to 2017. There are still many challenges to overcome regarding the diagnosis and management of TBM. First and foremost is patient selection for TBP. Most patients clearly experience dramatic relief in symptoms with a corresponding rise in PFTs. However, a minority of patients do not have significant benefit, but reasons are often unclear. Development of a prediction model may aid in selection when more data are available. Further investigation is needed to explicate the role of stents, optimal mesh size, and customized repair for unique malacic airway geometry. More advanced imaging may assist with operative planning and better noninvasive diagnosis. Although a minimally invasive approach may expand the options for patients with this complex disease, patient selection is still paramount and critical to optimal outcomes.

\section{Webcast}

You can watch a Webcast of this AATS meeting presentation by going to: https://aats.blob.core.windows.net/media/18Apr 30/25ABC\% 202.General\%20Thoracic \%20SS/S69\%20\%20Part\%201/S69_1_webcast_020125204.mp4.

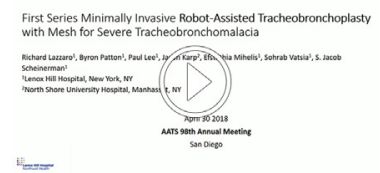

\section{Conflict of Interest Statement}

Authors have nothing to disclose with regard to commercial support.

\section{References}

1. Husta BC, Raoof S, Erzurum S, Mehta AC. Tracheobronchopathy from inhaled corticosteroids. Chest. 2017;152:1296-305.

2. Bhatt SP, Terry NL, Nath H, Zach JA, Tschirren J, Bolding MS, et al. Association between expiratory central airway collapse and respiratory outcomes among smokers. JAMA. 2016;315:498-505.

3. Ernst A, Rafeq S, Boiselle P, Sung A, Reddy C, Michaud G, et al. Relapsing polychondritis and airway involvement. Chest. 2009;135:1024-30.

4. Biswas A, Jantz MA, Sriram PS, Mehta HJ. Tracheobronchomalacia. Dis Mon. 2017; 63:287-302.

5. Carden KA, Boiselle PM, Waltz DA, Ernst A. Tracheomalacia and tracheobronchomalacia in children and adults: an in-depth review. Chest 2005; 127:984-1005. 
6. Stern EJ, Graham CM, Webb WR, Gamsu G. Normal trachea during forced expiration: dynamic CT measurements. Radiology. 1993;187:27-31.

7. Gangadharan SP, Bakhos CT, Majid A, Kent MS, Michaud G, Ernst A, et al. Technical aspects and outcomes of tracheobronchoplasty for severe tracheobronchomalacia. Ann Thorac Surg. 2011;91:1574-80.

8. Wright CD, Grillo HC, Hammoud ZT, Wain JC, Gaissert HA, Zaydfudim V, et al Tracheoplasty for expiratory collapse of central airways. Ann Thorac Surg. 2005; 80:259-66.

9. Buitrago DH, Wilson JL, Parikh M, Majid A, Gangadharan SP. Current concepts in severe adult tracheobronchomalacia: evaluation and treatment. J Thorac Dis. 2017;9:E57-66.

10. Nesbitt JC, Carrasco H. Expandable stents. Chest Surg Clin N Am. 1996;6:305-28.

11. Gangadharan SP. Tracheobronchomalacia in adults. Semin Thorac Cardiovasc Surg. 2010;22:165-73.

12. Lazar JF, Spier LN, Hartman AR, Lazzaro RS. Standardizing robotic lobectomy: feasibility and safety in 128 consecutive lobectomies within a single healthcare system. Innovations (Phila). 2017;12:77-81.

13. Emmert A, Straube C, Buentzel J, Roever C. Robotic versus thoracoscopic lung resection: a systematic review and meta-analysis. Medicine (Baltimore). 2017; 96:e7633.

14. Lazar JF, Posner DH, Palka W, Spier LN, Lazzaro RS. Robotically assisted bilateral bronchoplasty for tracheobronchomalacia. Innovations (Phila). 2015; 10:428-30.

15. Harris PA, Taylor R, Thielke R, Payne J, Gonzalez N, Conde JG. Research electronic data capture (REDCap)-a metadata-driven methodology and workflow process for providing translational research informatics support. J Biomed Inform. 2009; 42:377-81.

16. Dindo D, Demartines N, Clavien PA. Classification of surgical complications: a new proposal with evaluation in a cohort of 6336 patients and results of a survey. Ann Surg. 2004;240:205-13.

17. Jones PW, Quirk FH, Baveystock CM. The St George's Respiratory Questionnaire. Respir Med. 1991;85(Suppl B):25-31.

18. ATS Committee on Proficiency Standards for Clinical Pulmonary Function Laboratories. ATS statement: guidelines for the six-minute walk test. Am J Respir Crit Care Med. 2002;166:111-7.

19. Reddel HK, Taylor DR, Bateman ED, Boulet LP, Bourshey HA, Busse WW, et al. An official American Thoracic Society/European Respiratory Society statement: asthma control and exacerbations: standardizing endpoints for clinical asthma trials and clinical practice. Am J Respir Crit Care Med. 2009;180:59-99.

20. Celli BR, MacNee W, Force AET. Standards for the diagnosis and treatment of patients with COPD: a summary of the ATS/ERS position paper. Eur Respir J. 2004;23:932-46.

21. Jones PW. St George's Respiratory Questionnaire: MCID. COPD. 2005;2:75-9.

22. Hasegawa I, Boiselle PM, Raptopoulos V, Hatabu H. Tracheomalacia incidentally detected on CT pulmonary angiography of patients with suspected pulmonary embolism. AJR Am J Roentgenol. 2003;181:1505-9.

23. Wright CD, Edwards FH. Society of Thoracic Surgeons General Thoracic Surgery Database Task Force, Society of Thoracic Surgeons Workforce on National Databases. The Society of Thoracic Surgeons General Thoracic Surgery Database. Ann Thorac Surg. 2007;83:893-4.

Key Words: tracheobronchoplasty, tracheomalacia, bronchomalacia, tracheobronchomalacia, robotic, TBM, TBP, COPD

\section{Discussion}

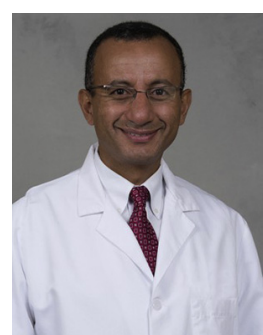

Dr Abbas Abbas (Philadelphia, Pa). Thank you, Dr Lazzaro. I want to commend you on the impressive series you and your coauthors have presented here. This study not only describes the first consistent minimally invasive modality to this operation of posterior tracheoplasty in adults, but also is among the largest series, period, for this operation. Perhaps among the most important findings in your study is the proof of concept that this difficult operation can be done consistently in a minimally invasive fashion and with safe and excellent results.

I agree with you that this disease is probably vastly underdiagnosed. With so many patients who potentially have severe tracheobronchomalacia, the value of tracheoplasty has already been established in other studies. However, these patients are often too frail or sick to undergo a lengthy thoracotomy and, therefore, the ideal operation would be a minimally invasive option. It is also not surprising that the entire Society of Thoracic Surgeons database has only 262 patients in a 15 -year period. You have done $20 \%$ of that number in just 18 months.

This brings me to my first question. I was impressed by the extremely high number of patients that your institution has screened for tracheobronchomalacia using dynamic computed tomography (CT) scan: 435 in 18 months. What was the impetus for this approach, which is certainly much higher than most other hospitals? Was it based on an institutional decision of your group to start actively screening for this disease?

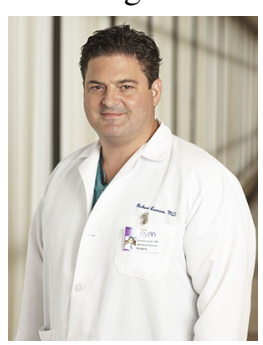

Dr Lazzaro (New York, NY). It was based on a case report. About 5 years ago we saw someone who met the criteria for tracheobronchoplasty and would not have been a candidate for an open approach and was declined open surgery. At that time we performed we used a minimally invasive approach, we reported it, it is several years later (4.5 years in fact), and he is well. That led to a conversation with a pulmonologist in our system. We work in New York, we have more than 10 million patients, we have 22 hospitals, and we have pulmonologists who have been in practice for more than 2 decades who have been able to take care of patients on a longitudinal basis. Over that time, they have recognized that their patients have become progressively symptomatic and less responsive to treatment. So a casual conversation about tracheobronchomalacia led to asking if I scan some patients who are falling down, who are clinically deteriorating, would you be willing to see them?

I don't think that any surgeon wakes up and says, this is the case that I want to do, but I was impressed with how impaired they were, and if we could replicate the excellent work of open tracheobronchoplasty, we might be able to further the adoption of it. So it's saying yes as opposed to no.

Dr Abbas. It seems that all patients with $>50 \%$ narrowing on dynamic $\mathrm{CT}$ were automatically referred for surgical evaluation. Why did your group choose $50 \%$ rather than $90 \%$ as the cutoff for referral?

Dr Lazzaro. There were studies done in the early 1990s that demonstrated a normal person has approximately a 
$34 \%$ reduction in the cross-sectional area during a bronchoscopy or dynamic CT scan. So if you used $>50 \%$ collapse, I think only 1 patient who was normal had that. So in general that is the definition of excessive dynamic airway collapse. Mild tracheobronchomalacia is $70 \%$ to $80 \%$ collapse, $80 \%$ to $90 \%$ is moderate tracheobronchomalacia, and severe tracheobronchomalacia is $>90 \%$.

When you do the dynamic CT scans, it is not necessarily on the forced exhalation, maximum exhalation that you see the maximum reduction in the cross-sectional area. So when we saw the screening tests demonstrate more than normal, we elected to evaluate them.

We didn't bronchoscope every patient. A patient needed to have clinical symptoms consistent with the radiographic diagnosis that would lead us down the path of a dynamic bronchoscopy.

Dr Abbas. So you found a discrepancy between the dynamic CT degree of narrowing and what you saw on bronchoscopy?

Dr Lazzaro. I can't say we found a discrepancy on all of the studies, but when you do a dynamic CT scan, the patients are coached several times and the technician has to capture the scan. So a dynamic CT can be very good to rule in, but if it's not captured at the right time of the expiratory cycle, it may underassess the severity of the airway collapse.

Dr Abbas. My final question is about the role of preoperative pulmonary function, which actually appeared only mildly depressed or close to normal. So in addition, the postoperative increase, although statistically significantly improved, was mild or modest. It doesn't seem that your group really placed much emphasis on the pulmonary function tests. So why did you use that as a parameter to check postoperatively rather than repeating a dynamic CT or bronchoscopy?

Dr Lazzaro. Although the dynamic CTs can be done with low radiation exposure, we use CT scans and bronchoscopies to evaluate patients with persistent symptoms. I think that's important. When I look at the literature of open tracheobronchoplasty, the goal has often been quoted as a patient who has symptomatically improved with an improvement in the quality of life. In fact, someone has even said that the holy grail is to obtain an objective measure of improvement. I think the peak expiratory flow is something that makes sense to me in terms of seeing an improvement when people have significant airway collapse. There are not many procedures available to thoracic surgeons. Relief of airway obstruction; decortication; and, at least in the short term, robotic tracheobronchoplasty can potentially improve pulmonary function tests.

So we did look at symptomatic improvement and, consistent with what is reported in the literature, have seen between $70 \%$ and $80 \%$ improvement in cough, the subjective feeling that the patient could breathe better and clear secretions better. I think as we are moving forward with our registry, we are looking at the improvement not only in pulmonary function but also in comorbid conditions. Very frequently, a patient will come back and say that his or her sleep apnea is better. Well, that makes sense, because it was probably intrathoracic as opposed to something cervical in the pharynx. We see that diabetes (anecdotally) and hypertension is better, and that might be just a reset of adrenal access and maybe they are no longer struggling to breathe. It has been a phenomenal several years of looking at this illness.

Dr Abbas. Thank you, Richard. I enjoyed your talk.

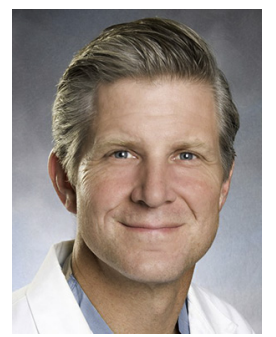

Dr Scott Swanson (Boston, Mass). Rich, that was phenomenal, really groundbreaking. Great results and nice work. Two quick questions: You may have mentioned this, but is there a learning curve and how steep is it? and, Do you have any insight into the complications that you are going to see or might see and how to avoid those if you start to really generalize this operation? I could imagine because this is going to change the way people recover from this and makes it easier that we could slide into doing this more easily for less-severe disease. In this meeting there is going to be a presentation on redos.

Dr Lazzaro. That's a great question. Thank you, Dr Swanson. I think there are technical aspects to the procedure that all of us in this room can do: dissect, cut, and sew. I think that not everybody has the same number of years or cases for robotic experience. I think really the key is to become facile with the robot so you can learn how to introduce the robot to give you the visualization to perform the open tracheobronchoplasties that Drs Grillo, Mathisen, Wright, Gangadharan, and Bueno all performed. Our learning curve is really not something where it's 20 procedures. I think that the learning curve was 5 years ago learning the robot.

When I look at our first 10 cases, we were really concerned about the placement of the sutures and not going full thickness, and then we gradually learned that you are magnified and you are able to see the width of your instrument and the width of the needle. The posterior membrane is 2- to 3- to 4-mm thick, and so you are placing those not from 18 in away, but tangentially in the same plane as the membrane. So it's just getting comfortable with a new approach to perform the same procedure.

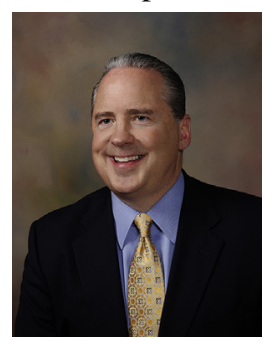

Dr Daniel L. Miller (Marietta, Ga). Rich, an incredible series. I see that you have abandoned preoperative stent placement, and that's what we have always used in our algorithm of doing these patients, because if the stent didn't improve them for whatever period of time, 10 to 14 days, we did not proceed. I just wonder why you have gone that route. 
Dr Lazzaro. I think there is no satisfactory stent. I think stent patients can tell you that they feel better, but they can get dislodged, obstructed, and cause more problems, and a negative stent trial does not necessarily mean that that patient won't improve. So it was a surrogate to see if someone improved. You would feel more justified in putting them through a posterior lateral thoracotomy with its associated risks.

Dr Miller. I was surprised about your lack of postoperative bronchoscopy requirement. Usually when we do them as an open technique, they have so much bronchorrhea that we are bronching them 2 or 3 times a day for that first 3 to 5 days, and you only had that occur once.

Dr Lazzaro. Our patients are sitting in a chair several hours postoperation and walking in the intensive care unit, frequently the chest tube is taken out a day later, and I think the facts that they did not have an epidural, they are not taking narcotics, and they are being managed with regional blocks administered in the operating room allows us to get them up and moving, and we really haven't seen that.

Dr Miller. Outstanding series. 